Pacific Journal of Mathematics

GENERALIZED HOMOTOPY EXCISION THEOREMS 


\title{
GENERALIZED HOMOTOPY EXCISION THEOREMS MODULO A SERRE CLASS OF NILPOTENT GROUPS
}

\author{
GRAHAM H. TOOMER
}

\begin{abstract}
We combine two well known homotopy equivalences of Ganea and some recent work on nilpotent spaces to give a common procedure for deriving the connectivity of generalized excision maps, given that the spaces involved are nilpotent rather than simply connected.
\end{abstract}

The main results are stated in the context of Serre classes of nilpotent groups. Our proof of the Blakers-Massey theorem appears to be new, and applies moreover to any map of nilpotent spaces which induces an epimorphism of fundamental groups (cf. [18, Corollary 6, p. 487]). We close with a very general Freudenthal Suspension Theorem.

M. Mather [16] has proved a theorem on mapping cones wich yields two basic weak homotopy equivalences of Ganea [9, Theorem 1.1] ([10, Theorem 1.1]) as special cases. In the first part of this paper we apply the former equivalence to study the excision maps related to a fibration, generalizing Serre's classical theorem. (The second part uses [10, Theorem 1.1] to derive a generalized BlakersMassey theorem related to a cofibration.) We have strived to emphasize a parallel treatment in the organization of this paper. Thus each part begins with a discussion of when the two generalized excision maps (associated to a fibration (cofibration)) are homomorphisms of nilpotent groups, in case $\mathscr{C} \neq\{0\}$ : see Corollary 2; and then we show that it is only necessary to determine the $\bmod \mathscr{C}$ connectivity of one of them: see Proposition 4. Ganea's equivalences are then used to derive the $\bmod \mathscr{C}$ connectivity of one of the associated maps: see Lemma 5. Finally we induct on the number of stages in a principal refinement of a Postnikov tower of a nilpotent space (the dimension of a finite $C W$ complex) to prove the general result: see Theorems I.12, II.7. (We chose a cellular argument over one using a homology decomposition as it avoids simple connectivity and is less technical.) The problem of finding a common procedure for deriving these excision theorems was raised in [15, p. 52].

We give a brief review of how our work is related to [1], [3], [11], and [17]. Associated to any space $B$ and a fibration $F \stackrel{j}{\rightarrow} Y \stackrel{p}{\rightarrow} X$, there is a transgression square 


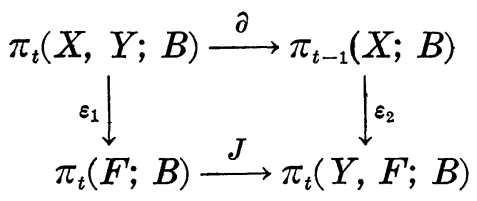

where $t \geqq 1,\left[15,\left(4.2^{\prime}\right)\right.$, p. 24]. Our proof of the $\bmod \mathscr{C}$ connectivity of $\varepsilon_{1}$ (when $t \geqq 2$ and $B=K(G, n)$ ) is an adaptation of [9, Proposition 2.1] to our context (cf. [1, Proposition 2.1].). For a given finite $C W$ complex $A$, a cofibration $X \stackrel{i}{\rightarrow} Y \stackrel{q}{\rightarrow} Y / X$ and $t \geqq 2$, [10, Theorem 1.1] is used to determine the $\bmod \mathscr{C}$ connectivity of $\pi_{t}(A ; Y, X) \rightarrow$ $\pi_{t}(A ; Y / X)$ when $A=S^{\circ}$. In the general case, we adapt [13, Theorem 6.1]. [11] and [1] were useful in translating this result into the "transgression square"

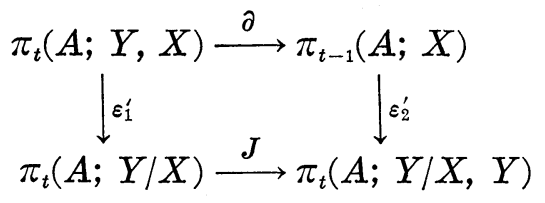

of $[11$, p. 82], where the case $t=1$ is studied in detail. See Theorem II.7 below (cf. 1, Theorem 2]). In [1], the Blakers-Massey theorem of [17] is used instead of [9]; for this reason, the hypotheses that $\pi_{1} X=0$ and $\pi_{2}(Y, X)=0$ are needed in [1, Theorem 2]. Also, we are not restricted to classes of finite abelian groups. Some generalized excision theorems modulo a class of finite abelian groups are also contained in [3], where spectral sequence techniques are used. We remark that in [11, Theorem p. 77], $B$ need only be nilpotent.

Combining our results with Lemma 1 (Lemma $1^{\prime}$ ) of [11], we now have a common procedure for the deriving of the connectivity of all generalized excision maps, basing ourselves on Lemma I.5 (Lemma II.5) (and taking $\mathscr{C}=\{0\}$ if $t=1$ ). Finally, each of these lemmas is in turn a consequence of Mather's result in [16] and standard arguments (if $\mathscr{C}=\{0\}$ ).

Conventions. All spaces, maps and homotopies will be based. $C X$ is the reduced cone on $X$, and $X \hookrightarrow C X$ is given by $x \mapsto[x, 0]$. $\Sigma X$ denotes the reduced suspension on $X . \quad P Y=\left\{\omega \in Y^{I} \mid \omega(0)=y_{0}\right\}$ and $P Y \rightarrow Y$ will always be $\omega \mapsto \omega(1)$.

The notation and conventions of [13], [14] will be used unless otherwise stated.

Homology will always mean reduced homology with integer coefficients.

I. Excision and fibrations. Let $F \stackrel{j}{\rightarrow} Y \stackrel{p}{\rightarrow} X$ be a fibration and 
$t \geqq 1$. For any space $B$, the commutative squares

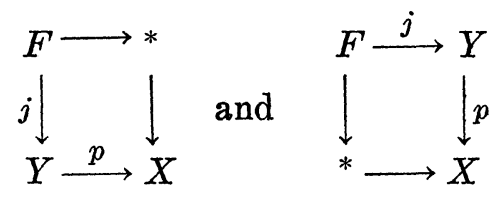

induce generalized excision maps $(p, *)_{\sharp}: \pi_{t}(X, * ; B) \rightarrow \pi_{t}(Y, F ; B)$ and $(*, j)_{\sharp}: \pi_{t}(X, Y ; B) \rightarrow \pi_{t}(*, F ; B),[15$, p. 13]. One shows that, for $t \geqq 2$, one is dealing with groups and homomorphisms as follows: Let $Z$ and $W$ be the pushouts defined by

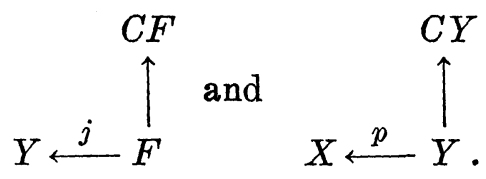

Let $\bar{p}: Z \rightarrow X$ extend $p$ by mapping $C F$ to the base point and let $i: \Sigma F \rightarrow W$ be the map naturally induced by $j$. It is well known that $(p, *)_{\sharp}$ and $(*, j)_{\sharp}$ may be identified with

$$
\bar{p}_{\sharp}:\left[X, \Omega^{t-1} B\right] \longrightarrow\left[Z, \Omega^{t-1} B\right]
$$

and

$$
i_{\sharp}:\left[W, \Omega^{t-1} B\right] \longrightarrow\left[\Sigma F, \Omega^{t-1} B\right] .
$$

See [9, Proposition 1.6].

We are interested in determing when $\bar{p}_{\#}$ and $i_{\sharp}$ are homomorphisms of nilpotent groups (when $t \geqq 2$ ).

Recall that a based space $\left(A, a_{0}\right)$ is said to have a nondegenerate base point if the inclusion $a_{0} \hookrightarrow A$ is an $h$-cofibration (i.e., $\left(A, a_{0}\right)$ has the homotopy extension property up to homotopy for all spaces). We say that $A$ is an amenable space if it is normal, Hausdorff, path connected and has a nondegenerate base point. Our first lemma is essentially due to Berstein and Ganea. We let nil $G$ denote the nilpotency class of a group $G$, and set nil $\{0\}=0$.

Lemma 1. Let $A$ be an amenable space and $B$ a space with nondegenerate base point. Then

$$
\text { nil }[A, \Omega B] \leqq \text { L.S. cat } A \text {. }
$$

(Here we set L.S. cat $\alpha_{0}=0$.)

Proof. It is well known that (i) since $B$ has a nondegenerate base point, so does $\Omega B$; and (ii) since $A$ has a nondegenerate base point $a_{0}, a_{0}$ will have a halo in $A$ which is contractible in $A$ to $a_{0}$. The result therefore follows from the inequalities of [4, Corollary 6.12]. 
CoRollary 2. Let $F \stackrel{j}{\rightarrow} Y \stackrel{p}{\rightarrow} X$ be a fibration of amenable spaces with $F$ and $Y$ countably paracompact, and let $B$ be any space with nondegenerate base point. If L.S. cat $X$, L.S. cat $Y<\infty$, then for $t \geqq 2$, $(p, *)_{\sharp}: \pi_{t}(X, * ; B) \rightarrow \pi_{t}(Y, F ; B)$ and $(*, j)_{\sharp}: \pi_{t}(X, Y ; B) \rightarrow \pi_{t}(*, F ; B)$ are homomorphisms of nilpotent groups.

Proof. Let $t \geqq 2$. As in the proof of Lemma $1, \Omega^{t-1} B=\Omega\left(\Omega^{t-2} B\right)$ will have a nondegenerate base point; here $\Omega^{\circ} B=B$. Identify $(p, *)_{\#}$ and $\bar{p}_{\sharp},(*, j)_{\sharp}$ and $i_{\sharp}$. We now check that $Z=Y \bigcup_{j} C F$ and $W=$ $X \cup_{p} C Y$ are amenable. By [7, Theorem 4], $F \times I$ and $Y \times I$ are normal. [2, Theorem 4.6.5] implies that $C F, C Y$ are Hausdorff and hence by [8, VII, 3.4] applied twice, $Y \cup C F$ (and $X \cup C Y$ ) are normal. Now [2, Theorem 4.6.5] easily implies that $Y \cup C F, X \cup C Y$ are Hausdorff. It is elementary that $Y \cup C F, X \cup C Y$ are path connected. Let $\left[y_{0}\right]$ denote the image of $y_{0} \in Y$ in $Y \cup C^{\prime} X$, where $C^{\prime} X$ is the unreduced cone on $X$. Using standard properties of $h$ cofibrations, it is easy to see that (i) $\left[y_{0}\right]$ is a nondegenerate base point for $X \cup C^{\prime} Y$ and (ii) the natural map $X \cup C^{\prime} Y \rightarrow X \cup C Y$ is a (based) homotopy equivalence. It follows that $X \cup C Y$ has a nondegenerate base point. Similarly, $Y \cup C F$ has a nondegenerate base point. Thus $Z$ and $Y$ are amenable. Finally, L.S. cat $Z \leqq$ L.S. cat $Y+1$, L.S. cat $W \leqq$ L.S. cat $X+1$ by [5, Theorem 2.6], and the result follows from Lemma 1.

Remarks. (i) Paracompact space and perfectly normal spaces are both examples of countably paracompact spaces. See [7].

(ii) The reader may verify that Corollary 2 also applies to the Serre fibration $\Omega X \rightarrow P X \stackrel{p}{\rightarrow} X$ when $X$ is an amenable space of the homotopy type of a simply connected $C W$ complex, and L.S. cat $X<$ $\infty$. In this case it is well known that $\left(p,{ }^{*}\right)_{\#}$ is also induced by the evaluation map $\Sigma \Omega X \rightarrow X$.

Definition 3. Let $B$ be a space, $\mathscr{C}$ a proper Serre class of nilpotent groups, $f: U \rightarrow V$ a map of connected spaces and $N \geqq 2$. We say that $(f ; B)$ is an $N$-equivalence $\bmod \mathscr{C}$ if

$$
\pi_{t}(f ; B): \pi_{t}(V ; B) \longrightarrow \pi_{t}(U ; B)
$$

is a homomorphism of nilpotent groups and $\pi_{t}(f, B)$ is a $\mathscr{C}$-surjection for $t>N$ and a $\mathscr{C}$-injection for $t \geqq N$ (in the sense of [13, Definition I.3]).

Proposition 4. Let $B$ and $F \hookrightarrow Y \rightarrow X$ satisfy the hypotheses of Corollary 2 and suppose that $(\bar{p} ; B):(Z ; B) \rightarrow(X ; B)$ is an N-equi- 
valence $\bmod \mathscr{C}$. Then $(i ; B):(W ; B) \rightarrow(\Sigma F ; B)$ is an $N$-equivalence $\bmod \mathscr{C}$.

Proof. By Corollary 2, we are dealing with homomorphisms of nilpotent groups. We have a ladder

$$
\begin{array}{r}
\pi_{t}(X ; B) \longrightarrow \pi_{t}(Y ; B) \stackrel{J}{\longrightarrow} \pi_{t}(X, Y ; B) \stackrel{\partial}{\longrightarrow} \pi_{t-1}(X ; B) \\
\pi_{t+1}(Y, F ; B) \stackrel{\partial}{\longrightarrow} \pi_{t}(Y ; B) \stackrel{J}{\left({ }^{*}, j\right) \sharp} \pi_{t}(F ; B) \stackrel{J}{\longrightarrow} \pi_{t}(Y, F ; B) \\
\longrightarrow \pi_{t-1}(Y ; B) \stackrel{J}{\longrightarrow} \pi_{t-1}(X, Y ; B) \\
\stackrel{\partial}{\longrightarrow} \pi_{t-1}(Y ; B) \longrightarrow \pi_{t-1}(F ; B)
\end{array}
$$

and for $t \geqq 2,(p, *)_{\sharp} \partial=-J(*, j)_{\sharp}$ by $\left[15\right.$, Theorem $\left.4.3^{\prime}\right]$. Invoking the " $\bmod \mathscr{C}$ four lemma" of [20], we easily deduce the result using our identification of $(p, *)_{\sharp}$ and $\bar{p}_{\sharp-1},(*, j)_{\sharp}$ and $i_{\sharp-1}$.

We remark that when $\mathscr{C}=\{0\}$, Proposition 4 is essentially [11, Proposition 1].

Lemma 5. Let $F \stackrel{j}{\rightarrow} Y \stackrel{p}{\rightarrow} X$ be a fibration and suppose that

(i) $F$ and $Y$ are path connected, and $X$ is simply connected.

(ii) $\mathscr{C}$ is a proper acyclic Serre class of abelian groups.

(iii) there are integers $m \geqq 1$ and $n \geqq 2$ such that $H_{r}(F) \in \mathscr{C}$ for $r \leqq m-1$ and $H_{s}(X) \in \mathscr{C}$ for $s \leqq n-1$.

(iv) $\mathscr{C}$ is complete or the homology of $F$ and $X$ is finitely generated in each dimension.

Then for any $t \geqq 2, \bar{p}_{\sharp}: \pi_{t-1}(Z) \rightarrow \pi_{t-1}(X)$ is $\mathscr{C}$-injective for $t<m+n$ and $\mathscr{C}$-surjective for $t \leqq m+n$.

Proof. Firstly, since $0=\pi_{1} X=\pi_{1}(Y, F) \rightarrow \pi_{1}(Y \cup C F), Z$ is simply connected and ker $\bar{p}_{\sharp}$ is abelian. By [9, Theorem 1.1] there is a weak homotopy equivalence $F * \Omega X \rightarrow \bar{p}^{-1}(*)=\bar{F}$, and hence an exact sequence

$$
[H(F) \otimes H(\Omega X)]_{t-2} \longleftrightarrow H_{t-1}(\bar{F}) \longrightarrow \text { Tor }[H(F), H(\Omega X)]_{t-3} .
$$

If $\mathscr{C}$ is not complete, (iv) and [18, Corollary 9.6.13] show that the homotopy groups of $X$ are finitely generated in each dimension. Now $\Omega X$ is nilpotent and so by [13, Theorem II.5.1] and [20, Proposition A.1], $H_{t-1}(\bar{F}) \in \mathscr{C}$ for $t-2 \leqq m+n-2$ under either of the hypotheses of (iv). Finally $\bar{F}$ is simply connected so that $\pi_{t-1}(\bar{F}) \in \mathscr{C}$ for $t-1 \leqq$ $m+n-1$. The exact homotopy sequence of $\bar{p}: X \rightarrow Z$ completes 
the argument.

We have given conditions which imply that $\bar{p}: Z \rightarrow X$ is an $N$ equivalence $\bmod \mathscr{C}$, and are interested in determining when for a given space $B,(\bar{p} ; B):\left[X, \Omega^{t-1} B\right] \rightarrow\left[Z, \Omega^{t-1} B\right]$ is a $\mathscr{C}$-surjection for $t>N^{\prime}$ and a $\mathscr{C}$-injection for $t \geqq N^{\prime}$ for some $N^{\prime}$. This necessitates the following definition.

DeFinition 6. Let $G$ be an abelian group. We say that a Serre class $\mathscr{C}$ of abelian groups is $G$-coacyclic if $C \in \mathscr{C} \Rightarrow \operatorname{Hom}(C, G) \in \mathscr{C}$ and $\operatorname{Ext}(C, G) \in \mathscr{C}$.

The next proposition explains why this notion can be used in conjunction with the universal coefficient theorem.

Proposition 7. Let $\varphi: A \rightarrow B$ be a homomorphism of abelian groups and $\mathscr{C}$ a G-coacyclic Serre class of abelian groups. Then

(i) coker $\phi \in \mathscr{C} \Rightarrow \operatorname{ker} \operatorname{Hom}\left(\varphi, 1_{G}\right) \in \mathscr{C}$.

(ii) $\operatorname{ker} \varphi \in \mathscr{C} \Rightarrow$ coker $\operatorname{Ext}\left(\varphi, 1_{G}\right) \in \mathscr{C}$.

(iii) if $\varphi$ is a $\mathscr{C}$-bijection, the same is true of $\operatorname{Hom}\left(\varphi, 1_{G}\right)$ and $\operatorname{Ext}\left(\varphi, \mathbf{1}_{G}\right)$.

Proof Since we are dealing with abelian groups, the usual sixterm exact sequence connecting Hom and Ext may be used.

It is time to give some examples of coacyclic classes:

REMARK 8. Let $G$ be any abelian group. The following Serre classes of abelian groups are G-coacyclic Serre classes. (In (ii)-(iv) we assume that $G$ is also finitely generated.)

(i) The class consisting of the trivial group.

(ii) The class of finitely generated abelian groups.

(iii) The class of finite abelian groups.

(iv) The class of finite $P$-torsion abelian groups, $P$ a multiplicative set of nonzero primes in $\boldsymbol{Z}$.

Proof. (i) is clear. To prove (ii)-(iv) one uses the structure theorem for finitely generated abelian groups and standard facts about Hom and Ext in a straightforward way.

Definition 9 ([2]). Let $G, H$ be groups and suppose that $G$ acts on $H$ in such a way that either (i) $G=H$ and $G$ acts on itself by conjugation or (ii) $H$ is abelian. Let $\Gamma_{G}^{1} H$ denote the subgroup of $H$ generated by elements of the form $(g \cdot h) h^{-1}$ where $g \cdot h$ denotes the action of $g \in G$ on $h \in H$. Set $\Gamma^{0} H=H$ and if $k \geqq 1, \Gamma_{G}^{k} H=\Gamma_{G}^{1}\left(\Gamma_{G}^{k-1} H\right)$. 
The action is nilpotent of class at most $c$ if $\Gamma_{G}^{k} H=0$ for $k \geqq c$. A path connected space $B$ is nilpotent if for each $n \geqq 1$, the action of $\pi_{1} B$ on $\pi_{n} B$ is nilpotent of class $c_{n}<\infty$. See [6, p. 58]. We say that a Serre class $\mathscr{C}$ is $B$-coacyclic if $\mathscr{C}$ is $\left(\Gamma^{i} \pi_{n} B / \Gamma^{i+1} \pi_{n} B\right)$-coacyclic for each $i \geqq 0$ and $n \geqq 1$.

REMARK 10. Suppose that $B$ is a nilpotent space and the homology of $B$ is finitely generated in each dimension. Then the homotopy groups of $B$ are finitely generated in each dimension by [13, Theorem 5.1] and thus each $\Gamma^{i} \pi_{n} B / \Gamma^{i+1} \pi_{n} B$ is finitely generated. Thus the examples (ii)-(iv) of Remark 8 are all $B$-coacyclic.

LEMMA 11. Suppose that $f: U \rightarrow V$ is a map of nilpotent spaces and let $B$ be a nilpotent $C W$ complex with $\pi_{i}(B)=0$ for $i \geqq d+1$. Let $\mathscr{C}$ be a proper Serre class of nilpotent groups such that the class of abelian groups in $\mathscr{C}$ is B-coacyclic. If $f: U \rightarrow V$ is an $N$ equivalence $\bmod \mathscr{C}$, then $(f ; B):(V ; B) \rightarrow(U ; B)$ is a $(d-N)$-equivalence $\bmod \mathscr{C}$.

Proof. We induct on the number $c$ of stages in a refinement of the Postnikov tower of $B$ into principal fibrations (see [12, Theorem 2.2.9]). The case $c=1$ means that $B=K(\pi, n), \pi$ abelian and $n \geqq 1$, so we can apply the Universal Coefficient Theorem [18, 5.3.3] and Proposition 7.

Suppose inductively that the result is true for any nilpotent $C W$ complex whose refinement has $\leqq c-1$ stages, $c \geqq 2$. Then we have a pullback

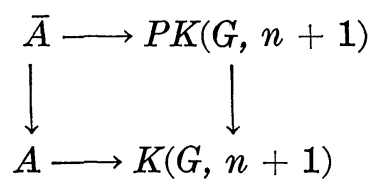

where $A$ is a nilpotent space with at most $c-1$ stages and $G=$ $\Gamma^{i} \pi_{n} \bar{A} / \Gamma^{i+1} \pi_{n} \bar{A}$ for some $i$ - see [12, Theorem II.2.9]. The long exact homotopy sequence of the fibration $\bar{A} \rightarrow A \rightarrow K(G, n+1)$, Proposition 7 and the "mod $\mathscr{C}$ four lemma" complete the argument, for $c<\infty$. The easy details are left to the reader.

THEOREM 12. Let $F \stackrel{j}{\hookrightarrow} Y \stackrel{p}{\rightarrow} X$ be a fibration of amenable spaces, with $F$ and $Y$ countably paracompact, L.S. cat $X$, L.S. cat $Y<\infty$.

Suppose that

(i) $F$ is nilpotent and $X$ is simply connected.

(ii) there are integers $m \geqq 1$ and $n \geqq 2$ such that $H_{r}(F) \in \mathscr{C}$ 
for $r \leqq m-1$ and $H_{s}(X) \in \mathscr{C}$ for $s \leqq n-1$, where $\mathscr{C}$ is a proper acyclic Serre class of nilpotent groups.

(iii) $\mathscr{C}$ is complete or the homology of $F$ and $X$ is finitely generated in each dimension.

(iv) $B$ is a nilpotent $C W$ complex satisfying

$$
\pi_{i}(B)=0 \text { for } i \geqq d+1 \text {. }
$$

(v) $\mathscr{C}^{\prime}$ is a B-coacyclic Serre class, where $\mathscr{C}^{\prime}$ denotes the (Serre) class of abelian groups in $\mathscr{C}$.

Then $(p, *)_{t}: \pi_{t}(X, * ; B) \rightarrow \pi_{t}(Y, F ; B)$ and $(*, j)_{t}: \pi_{t}(X, Y ; B) \rightarrow$ $\pi_{t}(*, F ; B)$ are $\mathscr{C}$-surjections for $t>d-m-n+2$ and $\mathscr{C}$-injective for $t \geqq d-m-n+2$.

Proof. By Corollary 2, $(p, *)_{t}$ and $(*, j)_{t}$ are homomorphisms of nilpotent groups. By Lemma $5, \bar{p}: Z \rightarrow X$ is an $(m+n-1)$-equivalence $\bmod \mathscr{C}$. Now $0=\pi_{1} X=\pi_{1}(Y, F) \rightarrow \pi_{1}(Y \cup C F)=\pi_{1} Z$ and so Lemma 11 may be applied to $\bar{p}: Z \rightarrow X$ with $N=m+n-1$. We deduce that $\bar{p}: Z \rightarrow X$ (and hence also $i: W \rightarrow \Sigma F$ by Proposition 4) is a $(d-m-n+1)$-equivalence $\bmod \mathscr{C}$. The result therefore follows from our identification of $(p, *)_{t}$ and $\bar{p}_{t-1},(*, j)_{t}$ and $i_{t-1}$.

We remark that, by considering $\Omega X \rightarrow P X \rightarrow X$ and $B=K(G, m)$, Theorem 12 yields results on the kernel and cokernel of the cohomology suspension map $H^{*}(X, G) \rightarrow H^{*-1}(\Omega X, G)$ where $X$ is a simply connected $C W$ complex of finite dimension. See Remark (ii) following Corollary 2 above.

II. Excision and cofibrations. Let $X \stackrel{i}{\rightarrow} Y \stackrel{q}{\rightarrow} Y / X$ be a cofibration and $t \geqq 1$. For any space $A$, the commutative squares

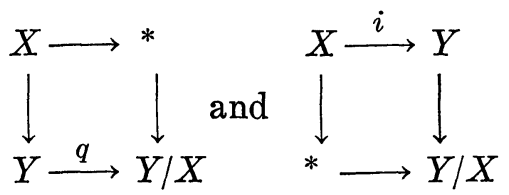

induce generalized excision maps $(q, *)_{\sharp}: \pi_{t}(A ; Y, X) \rightarrow \pi_{t}(A ; Y / X, *)$ and $(*, i)_{\sharp}: \pi_{t}(A ; *, X) \rightarrow \pi_{t}(A ; Y / X, Y)[15$, p. 13]. To see that we are dealing with groups and homomorphisms, let $Z$ and $W$ be the pullbacks defined by

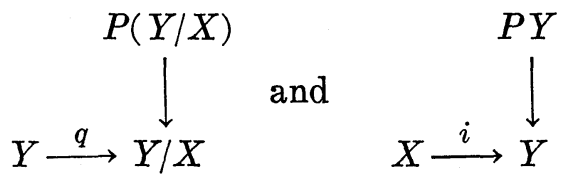


respectively. It is well known that there are natural maps $p: W \rightarrow$ $\Omega(Y / X)$ (induced by $q$ ) and $e: X \rightarrow Z$ (which is a lift of $i$ ) such that $(q, *)_{\sharp}$ and $(*, i)_{\sharp}$ may be identified with

$$
p_{\sharp}:\left[\Sigma^{t-1} A, W\right] \longrightarrow\left[\Sigma^{t-1} A, \Omega(Y / X)\right]
$$

and

$$
e_{\sharp}:\left[\Sigma^{t-1} A, X\right] \longrightarrow\left[\Sigma^{t-1} A, Z\right]
$$

respectively. (See [1, Lemma 3.7].)

We will use the following lemma to show that $e_{\sharp}$ and $p_{\ddagger}$ are often homomorphisms of nilpotent groups.

Lemma 1. Let $A$ be a finite $C W$ complex and let $B$ be any nilpotent space. Then the group $[\Sigma A, B]$ is nilpotent.

Proof. Let $B^{A}$ denote the path component of the unique constant map in the function space of based maps from $A$ to $B$. We can identify $[\Sigma A, B]$ and $\pi_{1}\left(B^{A}\right)$, and the latter group is nilpotent by [12, Corollary II.2.6].

REMARK. If $A$ is a connected $C W$ complex and $B$ has a non degenerate base point, we may use Lemma I.1 to deduce Lemma 1. The details are classical and familiar. Likewise for Corollary 2 below. Since nilpotency is required for Lemma 5 below anyway, we will not pursue this aspect.

Corollary 2. Let $X \stackrel{i}{\rightarrow} Y \stackrel{q}{\rightarrow} Y \mid X$ be a cofibration with $X$ and $Y$ nilpotent, $\pi_{1}(Y, X)=0$. If $A$ is a finite $C W$ complex, then

$$
\begin{aligned}
& (q, *)_{\sharp}: \pi_{t}(A ; Y, X) \longrightarrow \pi_{t}(A ; Y \mid X, *) \\
& (*, i)_{\sharp}: \pi_{t}(A ; *, X) \longrightarrow \pi_{t}(A ; Y / X, Y)
\end{aligned}
$$

are homomorphisms of nilpotent groups for $t>1$.

Proof. For $t \geqq 3$, all group are abelian. For $t=2$, we need only check that $Z, W$, and $Y / X$ are nilpotent spaces in view of our identification and Lemma 1 above. Since $X, Y$ are path connected and $\pi_{1}(Y, X)=0, \pi_{1}(Y / X)=0$. Thus $Z$ is path connected. Also, $\pi_{0}(W)=\pi_{1}(Y, X)=0$. Now $Z$ may be regarded as the fibre of a fibration $E \rightarrow Y / X$ where $E$ has the (based) homotopy type of $Y$, and hence by [12, II.2.2], $Z$ will be nilpotent. Similarly for $W . \quad \Omega(Y / X)$ is evidently nilpotent.

Definition 3. Let $f: U \rightarrow V$ be a map of nilpotent spaces, $N \geqq 2$, 
and $A$ a space. We say that $(A ; f)$ is an $N$-equivalence $\bmod \mathscr{C}$ if

$$
\pi_{t}(A ; f): \pi_{t}(A ; U) \longrightarrow \pi_{t}(A ; V)
$$

is a $\mathscr{C}$-injection for $1 \leqq t<N$ and a $\mathscr{C}$-surjection for $1 \leqq t \leqq N$, where $\mathscr{C}$ is a proper acyclic Serre class of nilpotent groups.

Proposition 4. Let $A$ and $X \stackrel{i}{\rightarrow} Y \stackrel{q}{\rightarrow} Y / X$ satisfy the hypotheses of Corollary 2. If $(A ; e):(A ; X) \rightarrow(A ; Z)$ is an $N$-equivalence $\bmod \mathscr{C}$, so is $(\mathrm{A} ; p):(A ; W) \rightarrow(A ; \Omega(Y / X))$.

Proof. Corollary 2 shows that $(A ; e)$ and $(A ; p)$ will induce homomorphisms of nilpotent groups.

The "mod $\mathscr{C}$ four lemma" of [20, Appendix] together with the homotopy ladder of [15, Theorem 4.3, p. 23] (and the identification prior to Lemma 1) easily yield the result. The details are dual to those of Proposition I.4 and are omitted.

Lemma 5. Suppose that (i) $X$ and $Y$ are nilpotent and $\pi_{1}(Y, X)=$ 0 ; (ii) $\mathscr{C}$ is complete or the homology of $X$ and $Y$ is finitely generated in each dimension; (iii) there are integers $m \geqq 1$ and $n \geqq 2$ such that $H_{r}(X) \in \mathscr{C}$ for $r \leqq m-1$ and $H_{s}(Y / X) \in \mathscr{C}$ for $s \leqq n-1$.

Then $e_{t-1}: \pi_{t-1}(X) \rightarrow \pi_{t-1}(Z)$ is a $\mathscr{C}$-injection for $t-1<m+n-2$ and $a \mathscr{C}$-surjection for $t-1 \leqq m+n-2$.

Proof. According to [10, Theorem 1.1], there is a weak homotopy equivalence $\Sigma\left(Z \cup_{e} C X\right) \rightarrow X * \Omega(Y / X)$, and the Künneth formula yields the exact sequence

$$
\begin{aligned}
{[H(X) \otimes H(\Omega(Y / X))]_{t-1} } & \longrightarrow H_{t-1}\left(Z \bigcup_{e} C X\right) \\
& \longrightarrow \text { Tor }[H(X), H(\Omega(Y / X))]_{t-2} .
\end{aligned}
$$

Now $Y / X$ is simply connected $H_{s}(Y / X) \in \mathscr{C}$ for $s \leqq n-1$. By [18, Corollary 11, p. 507], $H_{i}(\Omega(Y / X)) \in \mathscr{C}$ for $i \leqq n-2$. Also, $H_{s}(Y / X)$ is finitely generated for each $s \geqq 0$ if $X$ and $Y$ have finitely generated homology in each dimension. By [18, Corollary 13, p. 508] $H_{i}(\Omega(Y / X))$ is finitely generated for each $i \geqq 0$ in case $\mathscr{C}$ is not complete. The exact sequence above and $[20, \mathrm{~A} .1] \mathrm{imply}$ that $H_{t-1}\left(Z \mathrm{U}_{e} C X\right) \in \mathscr{C}$ for $t-1 \leqq m+n-2$. That is, $e_{t-1}: H_{t-1}(X) \rightarrow H_{t-1}(Z)$ is a $\mathscr{C}$ injection for $t-1<m+n-2$ and a $\mathscr{C}$-surjection for $t-1 \leqq$ $m+n-2$. The result now follows from [14, Theorem 3.4].

Our next result is the analogue of Lemma I.11, and the case $N=\infty$ is $[13,6.1]$. 
LEMMA 6. Let $f: U \rightarrow V$ be a map of nilpotent spaces, and let $A$ be a finite $C W$ complex of dimension $d$. If $f: U \rightarrow V$ is an $N$ equivalence $\bmod \mathscr{C}$, then $(A ; f):(A ; U) \rightarrow(A ; V)$ is an $(N-d)$-equivalence $\bmod \mathscr{C}$. (If $\mathscr{C}=\{0\}$, we can dispense with the requirement that $U$ and $V$ be nilpotent.)

Proof. This follows the method of [13, Theorem 6.1], using the "mod $\mathscr{C}$ four lemma" [20, Appendix] instead of the " $\bmod \mathscr{C}$ five lemma." Details are left to the reader.

THEOREM 7. Let $X \stackrel{i}{\rightarrow} Y \stackrel{q}{\rightarrow} Y / X$ be a cofibration and suppose that

(i) $X$ and $Y$ are nilpotent spaces such that $\pi_{1}(Y, X)=0$.

(ii) there are integers $m \geqq 1$ and $n \geqq 2$ such that $H_{r}(X) \in \mathscr{C}$ for $r \leqq m-1$ and $H_{s}(Y / X) \in \mathscr{C}$ for $s \leqq n-1$, where $\mathscr{C}$ is a proper acyclic Serre class of nilpotent groups.

(iii) $\mathscr{C}$ is complete or the homology of $X$ and $Y$ is finitely generated in each dimension.

(iv) $A$ is a finite $C W$ complex of dimension $d$. Then

$$
\begin{gathered}
(q, *)_{\sharp}: \pi_{t}(A ; Y, X) \longrightarrow \pi_{t}(A ; Y / X, *) \\
(*, i)_{\sharp}: \pi_{t}(A ; *, X) \longrightarrow \pi_{t}(A ; Y / X, Y)
\end{gathered}
$$

are $\mathscr{C}$-injections for $2 \leqq t<m+n-d-1$ and $\mathscr{C}$-surjections for $2 \leqq t \leqq m+n-d-1$.

Proof. This is analogous to the proof of Theorem 1.12: By Corollary 2, all groups in sight are nilpotent, and by Lemma 5, $e: X \rightarrow Z$ is an $(m+n-2)$-equivalence $\bmod \mathscr{C}$, and so Lemma 6 applies. We deduce that $(A ; e):(A ; X) \rightarrow(A ; Z)$ is an $(m+n-d-2)$ equivalence $\bmod \mathscr{C}$. The result now follows from our identification of $\left(q,{ }^{*}\right)_{t}$ and $p_{t-1},(*, i)_{t}$ and $e_{t-1}$ together with Proposition 4.

Some special cases of Theorem 7 are worth mentioning. The classical result (see e.g., [18, Corollary 6, p. 487]) is obtained by taking $A=S^{0}$ and $\mathscr{C}=\{0\}$ in Lemma 5. Notice that it is not necessary to have $\pi_{1}(X)=0$ and $\pi_{2}(Y, X)=0$. See also [19, Corollary 6.22]. When $\mathscr{C} \neq\{0\}$, and $n \geqq 3$ the case $A=S^{0}$ is implied by [14, Theorem 4.1]. For an application of Theorem 7, the reader may verify that in [15, Theorem 7.1'], $X$ need only be nilpotent.

CoROLLARY 8. Let $X$ be a nilpotent space and $m \geqq 1$ a fixed integer. If $H_{i}(X) \in \mathscr{C}$ for $i \leqq m-1$, where $\mathscr{C}$ is complete or the homology of $X$ is finitely generated in each dimension, then for any 
finite $C W$ complex $A$ of dimension $d<\infty$, the suspension homomorphism

$$
E:\left[\Sigma^{t-1} A, X\right] \longrightarrow\left[\Sigma^{t} A, \Sigma X\right]
$$

is a $\mathscr{C}$-injection for $2 \leqq t<2 m-d-1$ and a $\mathscr{C}$-surjection for $2 \leqq t \leqq 2 m-d-1$.

Proof. We apply Theorem 7 to $X \hookrightarrow C X \rightarrow \Sigma X$. It is easy to check that the lift " $e: X \rightarrow Z$ " of $X \hookrightarrow C X$ is the evaluation map $e: X \rightarrow \Omega \Sigma X$, and it is well known that $e$ induces the suspension homomorphism.

Now let $Q$ be a multiplicative set of primes in $Z$, and let $\mathscr{C}_{Q}$ be the acyclic Serre class of $Q$-torsion nilpotent groups. If we take $\mathscr{C}=\mathscr{C}_{Q}$ in Corollary 8 , we may delete the hypothesis that the homology of $X$ be finitely generated, for $\mathscr{C}_{Q}$ is complete. Thus Corollary 8 yields a localized version of the classical Freudenthal Suspension Theorem:

CoROLlary 9. Let $X$ be a nilpotent space and let $m \geqq 1$ be a fixed integer. If $H_{i}(X) \otimes \boldsymbol{Z}_{(P)}=0$ for $i \leqq m-1$ and $A$ is any finite $C W$ complex of dimension $d$, then

$$
E_{(P)}:\left[\Sigma^{t-1} A, X\right]_{(P)} \longrightarrow\left[\Sigma^{t} A, \Sigma X\right]_{(P)}
$$

is an injection for $2 \leqq t<2 m-d-1$ and a surjection for $2 \leqq t \leqq$ $2 m-d-1$.

Acknowledgments. I am indebted to Peter Hilton for sending me [11], [13], and [14], and for his interest. I also benefited greatly from his lectures on generalized Serre classes at the Ohio State University. Mary Ellen Rudin provided the precise reference needed for normality of mapping cones. The referee's helpful suggestions led to an improvement in the paper. To each, my sincere thanks.

\section{REFERENCES}

1. K. Ando, T. Kamagata, and K. Fukuhara, $O n \bmod \mathscr{C}$ excision theorems, Tôhuku Math. J., 25 (1973), 541-555.

2. R. Brown, Elements of Modern Topology, McGraw-Hill, New York (1968).

3. B. S. Brown, The mod $\mathscr{C}$ suspension theorem, Canad. J. Math., 21 (1969), 684-701.

4. I. Berstein and T. Ganea, Homotopical nilpotency, Illinois J. Math., 5 (1961), 99-130.

5. I. Berstein and P. J. Hilton, Category and generalized Hopf invariants, Illinois J. Math., 4 (1960), 437-451.

6. A. Bousfield and D. Kan, Homotopy limits, completions and localizations, Lecture Notes in Math. No. 304, Springer Verlag, Berlin, New York.

7. C. H. Dowker, On countably paracompact spaces, Canad. J. Math., 3 (1951), 219-224. 
8. J. Dugundji, Topology, Allyn and Bacon, Boston, Mass. (1960).

9. T. Ganea, A generalization of the homology and homotopy suspension, Comment. Math. Helvet., 39 (1965), 295-322.

10. On the homotopy suspension, Comment. Math. Helvet., 43 (1968), 225-234. 11. P. J. Hilton, On excision and principal fibrations, Comment. Math. Helvet., 35 (1961), 77-84.

12. P. J. Hilton, G. Mislin, and J. Roitberg, Localization of nilpotent groups and spaces, Amsterdam: North Holland. Mathematics Studies 15, 1975.

13. P. J. Hilton and J. Roitberg, Generalized C-theory and torsion phenomena in nilpotent spaces, Houston J. Math., 2 (1976), 525-559.

14. - On the Zeeman comparison theorem for the homology of nilpotent fibrations, Quarterly J. Math., 27 (1976), 433-444.

15. P. J. Hilton, Homotopy Theory and Duality, Gordon and Breach. New York (1965).

16. M. Mather, A generalization of Ganea's theorem on the mapping cone of the inclusion of a fibre, J. London Math. Soc., (2) 11 (1975), 121-122.

17. I. Namioka, Maps of pairs in homotopy theory, Proc. London Math. Soc., 12 (1962), 725-738.

18. E. H. Spanier, Algebraic Topology, McGraw-Hill, New York (1966).

19. R. M. Switzer, Algebraic Topology-Homotopy and Homology, Springer Verlag, Berlin, New York (1975).

20. G. H. Toomer, Homology equivalences and a technique of Ganea, Math. Z., 150 (1976), 273-279.

Received February 9, 1977 and in revised form May 9, 1977.

Ohio State University

Columbus, OH 43210 



\section{PACIFIC JOURNAL OF MATHEMATICS}

EDITORS

RICHARD ARENS (Managing Editor)

University of California

Los Angeles, California 90024

C. W. Curtis

University of Oregon

Eugene, OR 97403

C. C. MOORE

J. DUGUNDJI

Department of Mathematics University of Southern Californa Los Angeles, California 90007

R. Finn AND J. Milgram Stanford University Stanford, California 94305

University of California

Berkeley, CA 94720

\section{ASSOCIATE EDITORS}

E. F. BeCKENBACH

B. H. NeUMANN

F. WOLF

K. YosHida

\section{SUPPORTING INSTITUTIONS}

UNIVERSITY OF BRITISH COLUMBIA UNIVERSITY OF SOUTHERN CALIFORNIA CALIFORNIA INSTITUTE OF TECHNOLOGY UNIVERSITY OF CALIFORNIA MONTANA STATE UNIVERSITY UNIVERSITY OF NEVADA, RENO STANFORD UNIVERSITY UNIVERSITY OF TOKYO UNIVERSITY OF UTAH NEW MEXICO STATE UNIVERSITY WASHINGTON STATE UNIVERSITY OREGON STATE UNIVERSITY UNIVERSITY OF WASHINGTON UNIVERSITY OF OREGON OSAKA UNIVERSITY 


\section{Pacific Journal of Mathematics \\ Vol. 73, No. 1 \\ March, 1977}

Thomas Robert Berger, Hall-Higman type theorems. $V \ldots \ldots \ldots \ldots \ldots \ldots \ldots$

Frank Peter Anthony Cass and Billy E. Rhoades, Mercerian theorems via

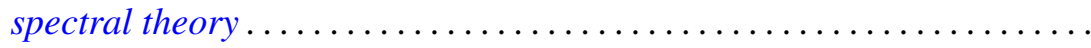

Morris Leroy Eaton and Michael David Perlman, Generating $\mathrm{O}(n)$ with

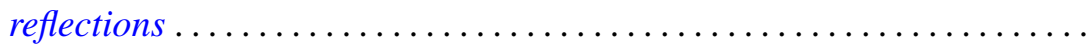

Frank John Forelli, Jr., A necessary condition on the extreme points of a

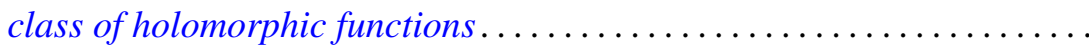

Melvin F. Janowitz, Complemented congruences on complemented

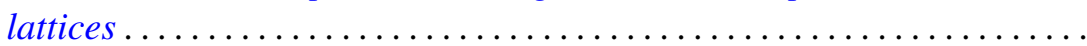

Maria M. Klawe, Semidirect product of semigroups in relation to amenability, cancellation properties, and strong $F \phi$ lner conditions....

Theodore Willis Laetsch, Normal cones, barrier cones, and the "spherical image" of convex surfaces in locally convex spaces ................

Chao-Chu Liang, Involutions fixing codimension two knots.............

Joyce Longman, On generalizations of alternative algebras .............

Giancarlo Mauceri, Square integrable representations and the Fourier

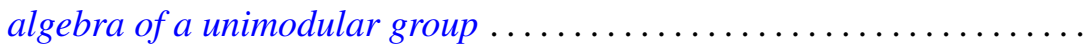

J. Marshall Osborn, Lie algebras with descending chain condition...

John Robert Quine, Jr., Tangent winding numbers and branched

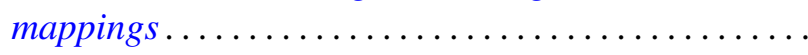

Louis Jackson Ratliff, Jr. and David Eugene Rush, Notes on ideal covers and associated primes .

H. B. Reiter and N. Stavrakas, On the compactness of the hyperspace of faces.

Walter Roth, A general Rudin-Carlson theorem in Banach-spaces ..

Mark Andrew Smith, Products of Banach spaces that are uniformly rotund in every direction.

Roger R. Smith, The R-Borel structure on a Choquet simplex ...

Gerald Stoller, The convergence-preserving rearrangements of real infinite series. ...

Graham H. Toomer, Generalized homotopy excision theorems modulo a Serre class of nilpotent groups...

Norris Freeman Weaver, Dehn's construction and the Poincaré conjecture....

Steven Howard Weintraub, Topological realization of equivariant intersection forms... 